\title{
Observation of strong magnetic effects in visible-infrared sum frequency generation from magnetic structures
}

\author{
A. Kirilyuk, G. M. H. Knippels, and A. F. G. van der Meer \\ FOM Institute for Plasma Physics Rijnhuizen, Edisonbaan 14, 3439 MN Nieuwegein, The Netherlands \\ S. Renard and Th. Rasing \\ Research Institute for Materials, University of Nijmegen, Toernooiveld 1, 6525 ED Nijmegen, The Netherlands \\ I. R. Heskamp and J. C. Lodder \\ MESA Research Institute, University of Twente, 7500 AE Enschede, The Netherlands
}

(Received 6 March 2000)

\begin{abstract}
We have observed very strong magnetization-induced changes of the infrared-visible sum-frequency generation (SFG) intensity from thin magnetic films using a free electron laser as a tunable infrared source. With the help of a magnetic grating a clear resonance is observed due to the excitation of surface plasmonpolaritons. The magnetization dependence of this resonance opens the way for nonlinear magneto-optical SFG studies of magnetic surface excitations.
\end{abstract}

In recent years, magnetization-sensitive second harmonic generation (MSHG) has been developed into a valuable method to study magnetic surfaces and multilayers. ${ }^{1-6}$ This nonlinear magneto-optical technique combines the convenience of an optical technique with intrinsic surface/interface sensitivity ${ }^{3,4}$ and strongly enhanced magneto-optical effects. ${ }^{5}$ For example, MSHG allowed to detect enhanced magnetic moments at atomic steps, ${ }^{7}$ to correlate interface roughness with interface anisotropy ${ }^{8}$ and to obtain very high contrast domain images for thin films. ${ }^{9}$ In addition, nonlinear magneto-optical effects have been discovered. ${ }^{10,11}$

Second harmonic generation is just a degenerate case of the general nonlinear optical process of three-wave mixing, $\omega_{1}+\omega_{2}=\omega_{3}$. The argument of symmetry breaking at interfaces, which yields the interface specificity of MSHG, should equally apply to magnetization-induced sumfrequency generation (MSFG). Though SFG has been used for surface studies for more than a decade, ${ }^{12,13}$ nothing is known on the magnetic properties of SFG. Compared to MSHG, MSFG would allow for much more spectroscopic opportunities, for example to probe magnetic excitations at surfaces and interfaces.

In this paper we demonstrate that such a generalization can indeed be carried out and leads to very strong $(>50 \%)$ effects on ultrathin films of iron on a GaAs(001) single crystal surface as well as on sandwiched $\mathrm{Pt} / \mathrm{CoNi} / \mathrm{Pt}$ layers and nanostructure arrays. By syncronizing the output of a frequency doubled Nd:YLF laser with the tunable IR output of a free electron laser we obtained an SFG signal that was strongly dependent on the magnetization. On an array of magnetic lines (magnetic grating) a clear resonance was observed as a function of the infrared wavelength, which is an indication of the excitation of surface plasmon-polariton modes. The dependence of this resonant feature on the magnetization direction suggests a considerable interaction of these modes with the magnetization.

The nonlinear optical polarization induced in a sample by two incident optical fields can be written as

$$
P_{i}^{(2)}\left(\omega_{s f g}\right)=\chi_{i j k}^{(2)}\left(\omega_{s f g} ; \omega_{i r}, \omega_{v i s}\right) E_{j}\left(\omega_{i r}\right) E_{k}\left(\omega_{v i s}\right),
$$

where $\omega_{s f g}, \omega_{i r}$, and $\omega_{v i s}$ are the frequencies of the SFG, infrared and visible beams, respectively, and $\chi_{i j k}^{(2)}$ is the second-order nonlinear optical susceptibility tensor similar as used for the description of MSHG. ${ }^{1}$ Both SHG and SFG are described by a third rank tensor that vanishes in media with inversion symmetry, yielding the sensitivity of both SHG and SFG for symmetry breaking interfaces. In the same way one can also immediately derive the nonzero $\chi_{i j k}^{(2)}$ elements that appear in the presence of a magnetization as they are the same for MSHG and MSFG. However, in the former case, the response is purely determined by the electronic density of states (DOS), for MSFG both the electronic as well as vibronic (phonons, magnons) states can contribute to the nonlinear optical response. While this is rather a practical limitation due to the measurement techniques, it is nevertheless a key point of interest to the SFG technique in general.

The direction in which this polarization radiates is found from the conservation of momentum parallel to the interface:

$$
k_{x}\left(\omega_{s f g}\right)=k_{x}\left(\omega_{v i s}\right)+k_{x}\left(\omega_{i r}\right) .
$$

The experiments were done at the Free-Electron Laser for Infrared eXperiments (FELIX) in Nieuwegein, The Netherlands, delivering tunable infrared radiation with wavelengths in the range of 5-240 $\mu \mathrm{m}$. This IR radiation comes in bursts, so-called macropulses, typically $5 \mu$ s long at a $5 \mathrm{~Hz}$ repetition rate. Each macropulse contains a large number of $1-3$ ps long micropulses, with a repetition rate of $1 \mathrm{GHz}$ that is given by the modulation frequency of the electron beam. The typical micropulse energy in the range of $5-50 \mu \mathrm{m}$ is $10 \mu \mathrm{J}$.

An actively mode-locked Nd:YLF oscillator is synchronized to FELIX in order to produce an SFG output. The laser is diode pumped and operates at exactly one quarter of the pulse repetition rate of FELIX (the actual frequency is $249.856650 \mathrm{MHz}$ ). It produces $6 \mathrm{ps}$ pulses with a typical 


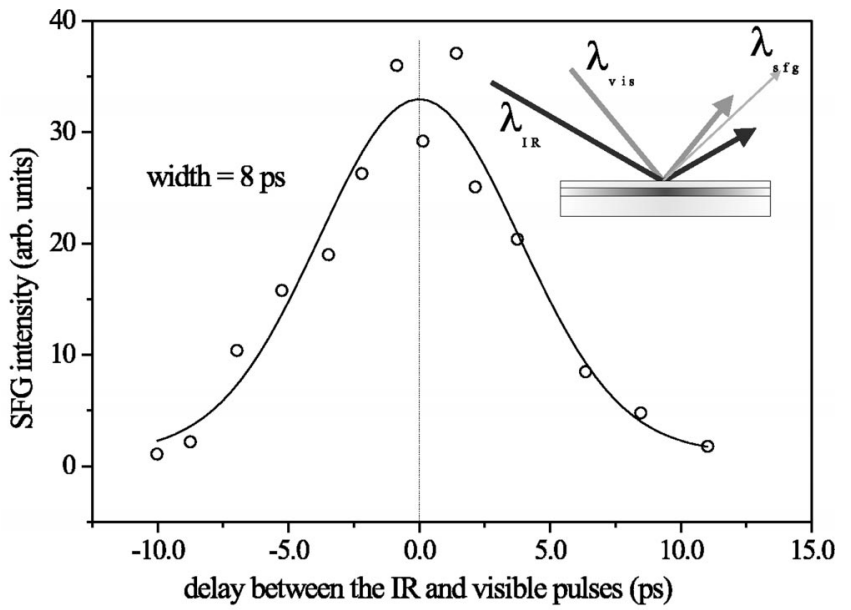

FIG. 1. SFG response from gold film as a function of the relative timing between the pulse trains of the Nd:YLF laser and FELIX. The inset shows the direction of the wave vectors of the incident and generated beams.

energy of $1 \mathrm{~nJ}$ at $1047 \mathrm{~nm}$. For proper mode-locking operation of the laser its cavity length is matched to the RF frequency that is applied to the mode-locker crystal, by actively controlling one cavity mirror with a piezo transducer. The remaining jitter was measured to be less than $1 \mathrm{ps}$. The delay between the two lasers was then adjusted with the help of an electronic phase shifter, allowing for delay changes of $1 \mathrm{~ns}$ maximum.

In order to obtain pulse energies comparable to those of FELIX the output of the Nd:YLF laser is amplified several orders of magnitude, then frequency doubled in a $\mathrm{KD}^{*} \mathrm{P}$ crystal, resulting in 7 ps pulses at $523.5 \mathrm{~nm}$ with an energy content up to $30 \mu \mathrm{J}$. The amplifier slicer was synchronized with the FELIX macropulses in order to obtain a similar time structure for both lasers.

Both lasers were only slightly focused (beam diameter of around $1 \mathrm{~mm}$ ) onto the sample in order to stay well below the damage threshold. The angles of incidence were $45^{\circ}$ and $53^{\circ}$ for the visible and infrared beams, respectively. The sample magnetization was perpendicular to their common plane of incidence, fixed with a magnetic field of up to $3 \mathrm{kOe}$ (transversal geometry, see inset in Fig. 1).

To detect the MSFG response, a back-illuminated liquid$\mathrm{N}_{2}$ cooled CCD camera was used. The high quantum efficiency (up to $70 \%$ at $500 \mathrm{~nm}$ ) and low background noise $(<1$ electron per pixel per hour) make such a camera eminently suited as a detector for the very weak SFG signals.

As a first set of test samples we used Fe layers $(10 \mathrm{~nm}$ thick) grown on GaAs(001) surface and covered by a $5 \mathrm{~nm}$ thick Au overlayer, that have been previously checked with MSHG. The known anisotropic behavior of the nonlinear magneto-optical response of this structure ${ }^{14}$ served as a reference for this pioneering study. On the other hand, to explore the spectral possibilities of the technique as well as the tunability of FELIX, we used a magnetic grating (period of 5 $\mu \mathrm{m})$ made of sputtered $\operatorname{Pt}(5 \mathrm{~nm}) / \mathrm{CoNi}(10 \mathrm{~nm}) / \operatorname{Pt}(40 \mathrm{~nm}) /$ $\mathrm{Si}(001)$ sandwiched layers. The properties of these $\mathrm{Pt} / \mathrm{CoNi}$ interfaces have also been studied before with the help of nonlinear magneto-optics. ${ }^{8}$

To test the synchronized operation of the Nd:YAG laser

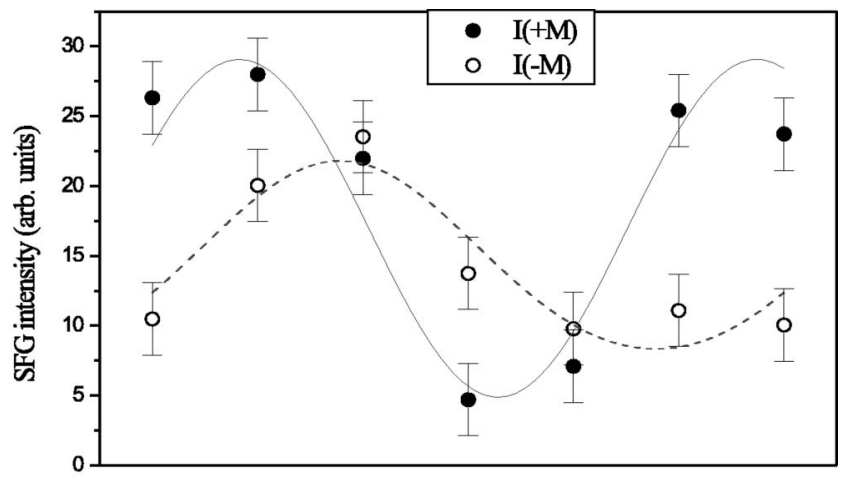

(a)

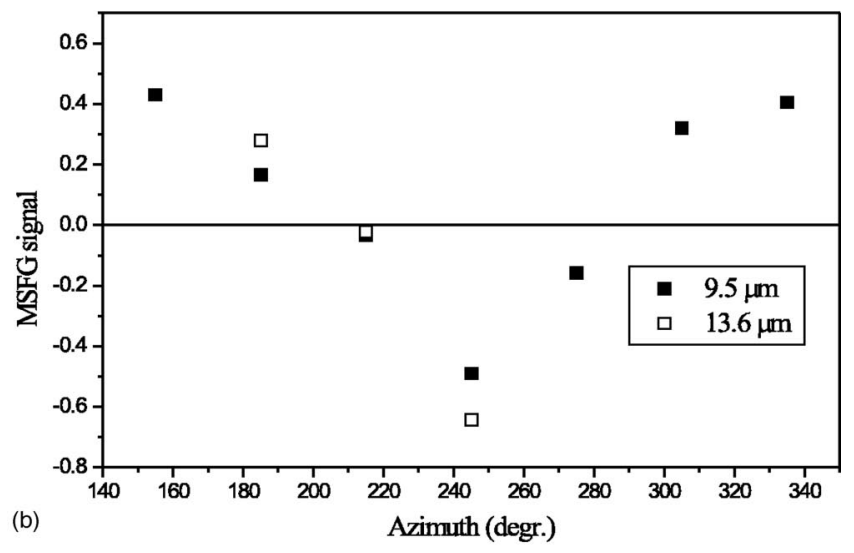

FIG. 2. SFG intensity for two opposite magnetization direction (a) and the magnetic asymmetry (b) as a function of the sample azimuthal angle measured in the $\mathrm{Au}(5 \mathrm{~nm}) / \mathrm{Fe}(10 \mathrm{~nm}) / \mathrm{GaAs}(001)$ sample with $\lambda_{I R}=9.5 \mu \mathrm{m}$. The solid lines represent the theoretical fit taking into account the twofold symmetry of the GaAs surface.

and FELIX, we measured the optical cross-correlation signal from a thick gold film on a glass plate. At $\lambda_{i r}=10 \mu \mathrm{m}$ the SFG response was recorded as a function of the relative time delay between the pulse trains of the two laser systems (see Fig. 1). A Gaussian fit through the recorded data gives a width of 8 ps FWHM. Given the duration of the visible $(\approx 7 \mathrm{ps})$ and infrared (1-2 ps) pulses, the measured crosscorrelation time indicates that the timing jitter is indeed rather small. It also indicates that there is no measurable drift of the pulse trains over the duration (several minutes) of such an experiment.

Figure 2(a) shows the SFG intensity as a function of the sample azimuthal angle for two opposite directions of $\mathbf{M}$, measured in the $\mathrm{Au}(5 \mathrm{~nm}) / \mathrm{Fe}(10 \mathrm{~nm}) / \mathrm{GaAs}(001)$ sample in the transversal magneto-optical geometry. The solid lines represent the theoretical fits of these dependencies by

$$
I^{s f g}( \pm M)=(A \sin 2 \varphi+B \pm C \cdot M)^{2},
$$

similar to that used in Ref. 14. Here, the azimuthal anisotropy is related to the crystallographic symmetry of the GaAs(001) surface.

The magnetization-induced change of the MSFG intensity is very large (up to more than 50\%), especially compared to what has been observed on the same samples with MSHG $(\leqslant 5-8 \%) .{ }^{14}$ It is not possible though to derive the reason for this difference from such singular measurement.

The magnetic asymmetry defined as 


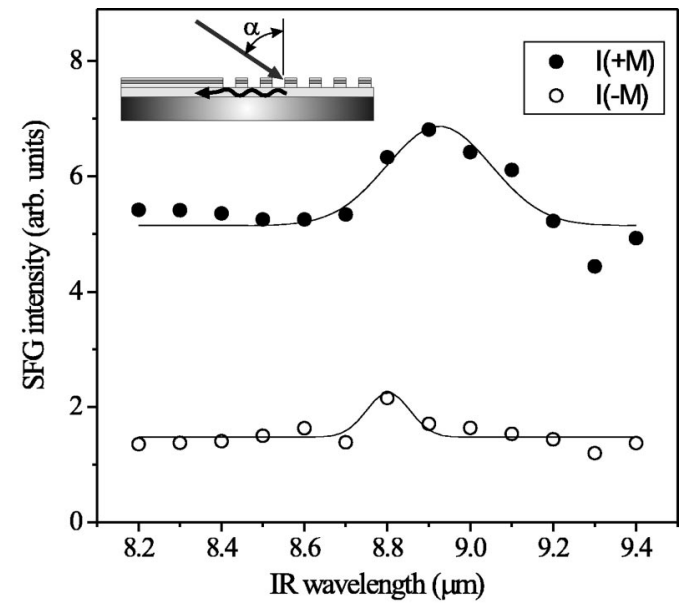

FIG. 3. SFG intensity for two opposite magnetization directions measured as a function of the FELIX wavelength on a $5 \mu \mathrm{m}$ grating made from $\mathrm{Pt} / \mathrm{CoNi} / \mathrm{Pt}$ sandwich.

$$
A=\frac{I^{s f g}(+M)-I^{s f g}(-M)}{I^{s f g}(+M)+I^{s f g}(-M)}
$$

is plotted in Fig. 2(b) for the two IR wavelengths, 9.5 and $13.6 \mu \mathrm{m}$. The slight increase of the asymmetry towards the longer wavelength is in line with the overall increase of $A$ from visible MSHG to the infrared-visible MSFG.

The access to the infrared region opens the possibility to study the interaction of the surface plasmon-polariton (SPP) modes ${ }^{15}$ with magnetization. In the visible, there is always a strong interference of these modes with interband transitions. However with photon energies below a few tenths of $\mathrm{eV}$ one obtains not only an effective excitation but also the propagation over macroscopic distances of surface plasmon polaritons. Because SPP modes are confined to the interface and propagate along its plane, they are particularly sensitive to the interface properties. In addition, due to a focusing effect, the field of an SPP can be considerably larger than the field used to excite it, leading to an enhancement of the nonlinear optical response. ${ }^{16}$

The excitation of an SPP by an incident $\mathrm{em}$ wave has to involve a coupling method that takes care of the excess of momentum carried by SPP. Here a grating with a period of $d=5 \mu \mathrm{m}$ is used for that purpose. For the effective excitation one has to choose the proper wavelength for a given angle of incidence. The wavevector of the SPP is given by

$$
K^{S P P}=k_{x}\left(\omega_{i r}\right) \pm k_{\text {grating }},
$$

with $k_{x}$ the component of the wavevector of the IR input radiation along the interface. For wavelengths longer than the grating period $d$ this leads to the following resonant condition (see also inset in Fig. 3):

$$
\lambda_{\text {res }}^{i r}=d \cdot(1+\sin \alpha)
$$

which yields $\lambda_{\text {res }}^{i r}=8.99 \mu \mathrm{m}$ for $\alpha=53^{\circ}$.

Figure 3 shows the SFG intensity as a function of the infrared wavelength measured on the $\mathrm{Pt} / \mathrm{CoNi} / \mathrm{Pt}$ grating, where a clear resonance is observed at $\lambda=8.9 \mu \mathrm{m}$ in strict agreement with Eq. (6). The increase of the SFG intensity is a consequence of the buildup of the interface field due to the excitation of the SPP's. The observed resonance can be fitted by the Lorentzian

$$
\chi^{(2)}(\omega)=\frac{a}{\omega_{S P P}-\omega-i \Gamma}+b
$$

where $a$ and $b$ are constants indicating the relative weights of the resonant and nonresonant contributions, $\omega_{S P P}=139 \mathrm{meV}$ and $\Gamma=2.5 \mathrm{meV}$ for $I(+M)$. For the opposite magnetization direction, very small resonant signal does not allow for any numbers to be derived from the data. Figure 3 not only shows that SFG from SPP's is feasible but also that the observed resonance is strongly affected by the magnetization. This opens the way to study surface spin excitations. By changing the spatial overlap between the IR and visible beam, the damping of the SPP and its magnetization dependence can directly be measured.

Note that the detector does not capture all SFG radiation that is generated at the sample: due to the presence of the grating, the SFG couples into many diffraction orders because the grating constant $d \gg \lambda_{s f g}$. Of course, it would also be of interest to study the other orders as well, especially as a function of the magnetization direction.

To conclude, we have demonstrated the feasibility of magnetization-induced sum frequency generation and in this way further extended nonlinear magneto-optics into the infra-red regime. Similar to the previously studied MSHG, this MSFG shows a strong dependence on the sample magnetization and thus yields a way to study low energy magnetic surface excitations. We have illustrated these possibilities with MSFG experiments on a magnetic grating of a $\mathrm{CoNi} / \mathrm{Pt}$ structure, that showed an enhancement of the MSFG intensity due to the excitation of a surface plasmon-polariton. Finally, we have also shown that the FELIX free electron laser appears to be a reliable and convenient source for the nonlinear (magneto-) optical experiments in the middle- and far-infrared.

We gratefully acknowledge the support by the Stichting voor Fundamenteel Onderzoek der Materie (FOM) in providing the required beam time on FELIX and highly appreciate the skillful assistance by the FELIX staff. Part of this work was supported by the EU network NOMOKE.
${ }^{1}$ Ru-Pin Pan, H.D. Wei, and Y.R. Shen, Phys. Rev. B 39, 1229 (1989).

${ }^{2}$ J. Reif, J.C. Zink, C.M. Schneider, and J. Kirschner, Phys. Rev. Lett. 67, 2878 (1991).

${ }^{3}$ H.A. Wierenga, W. de Jong, M.W.J. Prins, Th. Rasing, R. Voll- mer, A. Kirilyuk, H. Schwabe, and J. Kirschner, Phys. Rev. Lett. 74, 1462 (1995).

${ }^{4}$ M. Straub, R. Vollmer, and J. Kirschner, Phys. Rev. Lett. 77, 743 (1996).

${ }^{5}$ B. Koopmans, M. Groot Koerkamp, Th. Rasing, and H. van den 
Berg, Phys. Rev. Lett. 74, 3692 (1995).

${ }^{6}$ T.M. Crawford, C.T. Rogers, T.J. Silva, and Y.K. Kim, Appl. Phys. Lett. 68, 1573 (1996).

${ }^{7}$ Q.Y. Jin, H. Regensburger, R. Vollmer, and J. Kirschner, Phys. Rev. Lett. 80, 4056 (1998).

${ }^{8}$ A. Kirilyuk, Th. Rasing, M.A.M. Haast, and J.C. Lodder, Appl. Phys. Lett. 72, 2331 (1998).

${ }^{9}$ A. Kirilyuk, V. Kirilyuk, and Th. Rasing, J. Magn. Magn. Mater. 198-199, 620 (1999).

${ }^{10}$ V.V. Pavlov, R. V. Pisarev, A. Kirilyuk, and Th. Rasing, Phys. Rev. Lett. 78, 2004 (1997).

${ }^{11}$ A. Kirilyuk, V.V. Pavlov, R.V. Pisarev, and Th. Rasing, Phys. Rev. B 61, 3796 (2000).
${ }^{12}$ X.D. Zhu, H. Suhr, and Y.R. Shen, Phys. Rev. B 35, 3047 (1987); P. Guyot-Sionnest, J.H. Hunt, and Y.R. Shen, Phys. Rev. Lett. 59, 1597 (1987).

${ }^{13}$ A.L. Harris, C.E.D. Chidsey, N.J. Levinos, and D.N. Loiacono, Chem. Phys. Lett. 141, 350 (1987).

${ }^{14}$ S. Renard, A. Kirilyuk, A.M. Keen, Th. Rasing, T. Zhang, and T.-H. Shen (unpublished).

${ }^{15}$ Surface Polaritons, edited by V.M. Agranovich, and D.L. Mills (North-Holland, Amsterdam, 1982).

${ }^{16}$ E.V. Alieva, Y.E. Petrov, V.A. Yakovlev, V.A. Sychugov, E.R. Eliel, E.W.M. van der Ham, Q.H.F. Vrehen, and A.F.G. van der Meer, Pis'ma Zh. Éksp. Teor. Fiz. 66, 581 (1997) [JETP Lett. 66, 609 (1997)]. 